\title{
Characterization of plasma in a Hall thruster operated at high discharge voltage
}

\author{
Yevgeny Raitses*, Artem Smirnov ${ }^{\dagger}$, David Staack ${ }^{\star}$ \\ Princeton University Plasma Physics Laboratory, Princeton, NJ, 08543 \\ Michael Keidar ${ }^{\S}$ \\ University of Michigan, Ann Arbor, MI 48109 \\ and \\ Nathaniel J. Fisch ${ }^{\text {*** }}$ \\ Princeton University Plasma Physics Laboratory, Princeton, NJ, 08543
}

The electron-wall interaction and its dependence on the discharge voltage and channel width are studied through measurements of the electron temperature, plasma potential and density in a $2 \mathrm{~kW}$ Hall thruster. Experimental results are compared with theoretical predictions for different thruster configurations and operating conditions. The channel width is shown to have a more significant effect on the axial distribution of the plasma potential than the discharge voltage.

\section{Nomenclature}

$v_{e w} \quad=$ electron-wall collision frequency

$\Gamma_{e w} \quad=$ flux of primary electrons to the walls

$n_{e} \quad=$ plasma density

$h=$ distance between the walls of the annular channel (channel width)

$T_{e} \quad=$ electron temperature

$M_{i} \quad=$ ion mass

$\delta=$ secondary electron emission coefficient of the wall material

$\Gamma_{\text {esec }}=$ flux of secondary electrons from the wall

$\varepsilon_{w} \quad=$ energy loss at the wall per one electron

$\phi_{w} \quad=$ voltage drop between the plasma and the wall

\section{Introduction}

$\mathrm{H}$ igh-Isp applications for the Earth orbiting and interplanetary space missions require operation of Hall thrusters at high discharge voltages. For conventional Hall thrusters (so-called stationary plasma thrusters ${ }^{1,2}$ (SPT)) this requirement further intensifies scientific and technological challenges associated with the effective control the plasma flow in these cross-field discharge devices. The plasma-wall interaction and magnetic insulation properties of the thruster discharge can be strongly affected by the discharge voltage. In high discharge voltage regimes, a strong secondary electron emission (SEE) from ceramic channel walls may increase the electron-wall collision frequency, leading to the increase in the power losses. ${ }^{1-4}$ Along with it, SEE-induced near-wall conductivity is predicted to enhance electron cross-field transport ${ }^{1,2}$ and, thereby, reduce the accelerating electric field inside the

\footnotetext{
${ }^{*}$ Research Physicist, PPPL, P. O. Box 451, Princeton, NJ 08543, AIAA member.

${ }^{\dagger}$ Graduate Student, PPPL, P. O. Box 451, Princeton, NJ 08543.

¥ Graduate Student, PPPL, P. O. Box 451, Princeton, NJ 08543.

${ }^{\S}$ Research Professor, Aerospace Engineering, University of Michigan, Ann Arbor, MI 48109, AIAA member.

** Professor, PPPL, P. O. Box 451, Princeton, NJ 08543, AIAA member.
} 
thruster channel. ${ }^{5}$ As a consequence of the electric field reduction, the ion beam divergence can become large, increasing the channel wall erosion and making difficult the integration of the thruster with a satellite.

In recent experiments at PPPL, ${ }^{6,7}$ we studied macroscopic effects of the Joule heating and the electron-wall interaction on the plasma properties in a $2 \mathrm{~kW}$ Hall thruster. In this paper, we highlight several key implications of these studies for high discharge voltage operation of Hall thrusters. In Ref. 7 we compared experimental results with the predictions of existing fluid models of the electron-wall interaction in Hall thrusters. In this paper, we further demonstrate that the main conclusions of Ref. 7 on the role of SEE in high discharge voltage thruster operation are valid for different thruster configurations and operating conditions. In addition, we review the channel narrowing effect $^{6}$ on the plasma potential distribution, which, for some applications, may be an acceptable way to achieve a longer thruster lifetime. For the purpose of this review, we present additional results of thruster and plasma measurements at high discharge voltage operation. The paper is organized as follows. Section II presents in brief the commonly accepted model of electron-wall interaction in Hall thrusters. Sections III and IV describe experimental setup and thruster operation, respectively. Experimental results are analyzed in Section IV.

\section{Electron-Wall Interaction}

In a typical Hall thruster, the electron temperature is in the range $20-50 \mathrm{eV}{ }^{8,9}$ For the Maxwellian electron energy distribution function (EDF), the electron temperature within this range is sufficiently large to induce strong secondary electron emission (SEE) from ceramic channel walls. ${ }^{10}$ In the commonly accepted model of the electronwall interaction, ${ }^{2,4,10,11}$ the electron-wall collision frequency and the electron energy loss at the walls for the Maxwellian electrons can be expressed as

$$
\begin{aligned}
& v_{e w} \equiv \frac{\Gamma_{e w}}{n_{e} h} \approx \frac{1}{h} \sqrt{\frac{T_{e}}{M_{i}}} \frac{1}{1-\delta\left(T_{e}\right)}, \\
& v_{e w} \varepsilon_{w}=\frac{\tilde{v}}{h} \sqrt{\frac{T_{e}}{M_{i}}} \frac{1}{1-\delta\left(T_{e}\right)}\left(2 T_{e}+\left(1-\delta\left(T_{e}\right)\right) e\left|\phi_{w}\right|\right),
\end{aligned}
$$

respectively, where $\tilde{v} \sim 0.7-1.2$ for typical thruster conditions. ${ }^{3}$ Here, $\delta \equiv \Gamma_{\text {esed }} / \Gamma_{\text {ew }}$. The flux of primary electrons from the plasma to the floating wall is balanced by the flux of ions from the plasma and the flux of secondary electrons from the wall. When the SEE coefficient reaches approximately 1, the near-wall sheath becomes space charge saturated (SCS). ${ }^{12}$ Any further increase of the secondary electron flux into the plasma is restricted by a potential minimum formed near the wall surface. Under such conditions, the channel wall acts as an extremely effective energy sink (Eq. (2)), which tends to limit the electron temperature. ${ }^{2-4}$

According to Eq. (1), the electron-wall collision frequency depends on the electron temperature, SEE properties of the channel wall material and the channel width. The electron temperature depends on the Joule heating, which can be controlled by the discharge voltage. We varied the parameters of Eq. (1) and compared the experimental results with simulations.

\section{Experimental Setup}

In these experiments a $2 \mathrm{~kW}$ laboratory Hall thruster with boron nitride (grade HP) channel walls was used as a benchmark thruster configuration (Fig. 1). The channel width of this thruster is $25 \mathrm{~mm}$. The thruster, facility and diagnostics used in these experiments are described elsewhere. ${ }^{6,7,9,13,14}$ In order to change the channel width two boron nitride spacers were added to the inner and outer channel walls of the wide channel (Fig. $1 \mathrm{~b}$ ). With each spacer of $5 \mathrm{~mm}$ thick, the width of this channel became $15 \mathrm{~mm}$. Because the magnetic field was fixed, the insertion of these ceramic spacers reduced the magnetic mirror ratio near the inner wall (Fig. 1b) from 2.1 to $1.5{ }^{6}{ }^{6}$ Therefore, when we refer to the channel narrowing it includes also the reduction of the mirror near the inner wall.

The thruster experiments took place in a $28 \mathrm{~m}^{3}$ vacuum vessel equipped with cryogenic pumps. In each configuration, the thruster was operated at a constant xenon mass flow rate of about $2 \mathrm{mg} / \mathrm{s}$ and a fixed magnetic field. The background pressure did not exceed $6 \mu$ torr. We used two different magnetic field topologies for each thruster configuration. The plasma potential, electron temperature, and plasma density were measured along the channel median by employing a fast movable setup ${ }^{6,7,14,15}$ with low disturbing shielded probes. ${ }^{15}$ The plasma potential and electron temperature were deduced from the floating emissive and cold probe measurements, while the plasma density was measured with a biased cylindrical probe. The measurement procedure and analysis of physical uncertainties of the probe measurements are described in detail in our recent paper. ${ }^{7}$ 
(a)

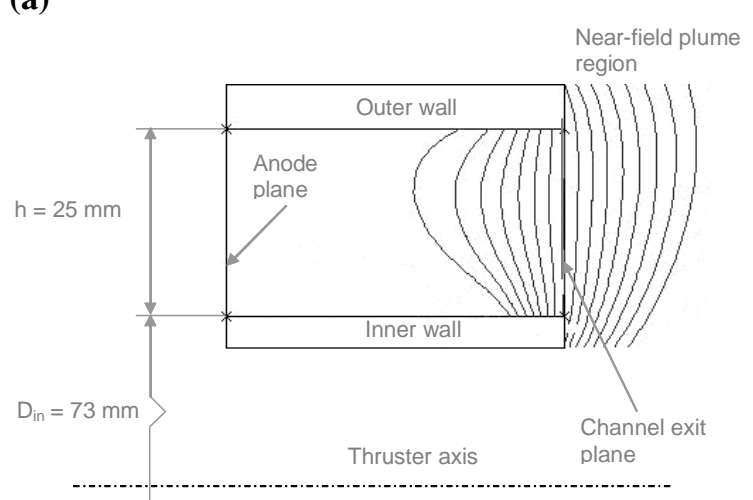

(b)

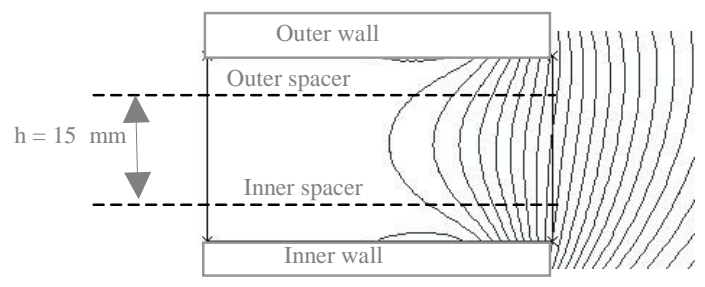

Figure 1. Schematic of the thruster channel with superimposed magnetic field lines for the benchmark thruster configuration ${ }^{6,7}$ (a) and for the thruster configuration with two BN ceramic spacers ${ }^{6}(b)$.

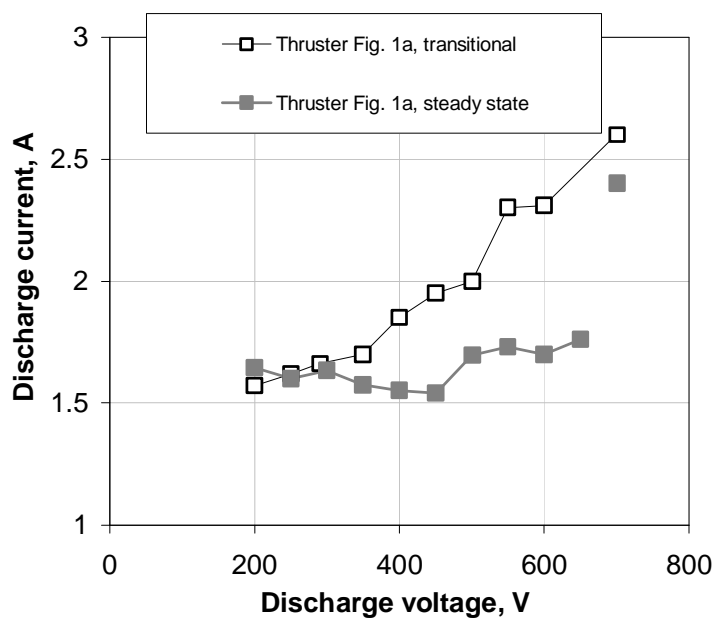

Figure 2. Discharge characteristics of a $2 \mathrm{~kW}$ Hall thruster in steady state and transitional regimes at the xenon gas flow rate (anode) of $19 \mathrm{sccm}$. The magnetic field is not changed with the discharge voltage (Fig. 1a).

In addition, the angular distribution of the ion flux from the thruster was measured with a 25-mmdiamete planar guarding sleeve probe ${ }^{16}$ (often called "the Faraday probe" in thruster publications). The plume probe was placed at the distance of $700 \mathrm{~mm}$ from the channel exit and rotated $\pm 90^{\circ}$ relative to the thruster axis. ${ }^{14}$ Both the probe and the sleeve were biased $-30 \mathrm{~V}$ with respect to ground. In addition to the fast movable biased probe, the plasma density distribution was also computed from the plume measurements outside the channel exit and plasma potential measurements inside the channel.

\section{A Few Remarks on Thruster Operation}

At the beginning of each set of the measurements, in order to reach a steady state the thruster was continuously operated at the discharge voltage of 200 $-300 \mathrm{~V}$ for approximately 1-2 hours. The steady state was monitored by measuring the discharge current. Then the discharge voltage was changed to the desired operating level and the probe measurements were performed. When increasing the voltage to 350$400 \mathrm{~V}$ and higher we encountered two steady state regimes of the thruster (Fig. 2), one of which appears to be transitional. ${ }^{17,18}$ The discharge characteristics measured in transitional and steady state operating regimes are given in Ref. 13. During the transitional operation, the discharge current is always larger than the steady state value. In Refs. 6 and 7, we compared measured plasma properties of the thruster discharge in these operating regimes. It appears that the main conclusions of these studies on the role of SEE in high discharge voltage thruster operation are valid for both transitional and steady state regimes.

\section{Experimental Results}

The effects of the discharge voltage on the electron temperature were studied for different magnetic field and channel width configurations. The electron temperature has a local maximum, which is in the vicinity of the high potential side of the acceleration region. $^{6-9}$ Fig. 3 shows how the maximum electron temperature, $T_{\mathrm{emax}}$, depends on the discharge voltage. Within the accuracy of probe measurements, the dependence of $T_{\text {emax }}$ on the discharge voltage exhibits fairly similar general trends for different magnetic field and channel width configurations, and for both transitional and steady state regimes (Fig. 3a). The discharge voltage threshold, $V_{\mathrm{d}} \sim 400 \mathrm{~V}$, separates two temperature regimes: i) below the voltage threshold $T_{\text {emax }}$ increases almost linearly with the discharge voltage and ii) in the range $400 \mathrm{~V}<\mathrm{V}_{\mathrm{d}}<600 \mathrm{~V}$, the electron temperature saturates at about $50-60 \mathrm{eV}$. Some increase of $T_{\text {emax }}$ is also observed at $V_{\mathrm{d}}>550-600 \mathrm{~V}$, which may manifest a transition to the third temperature regime. 
The dependence of the maximum electron temperature on the discharge voltage seemingly agrees with the theoretical predictions of Refs. 2-4. The fluid models ${ }^{2-4}$ explain the temperature saturation as a transition to the SCS regime of the near-wall sheaths. However, in the experiment, the saturation temperature exceeds the critical value, estimated under the assumption of the Maxwellian electron EDF, ${ }^{10,22}$ by a factor of about 3 . In addition, the discharge voltage threshold appears to be almost two times higher than that predicted by the fluid model ${ }^{3,4}$ for a lower SEE wall material.

(a)

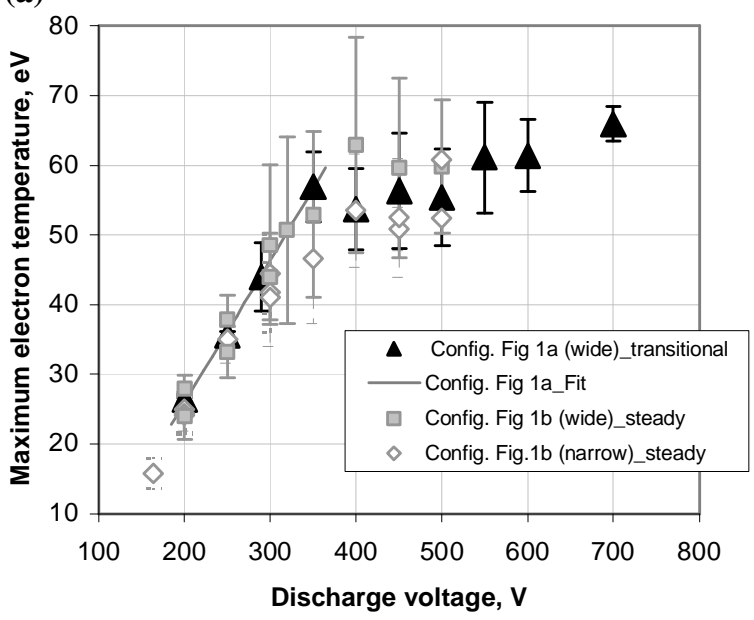

(b)

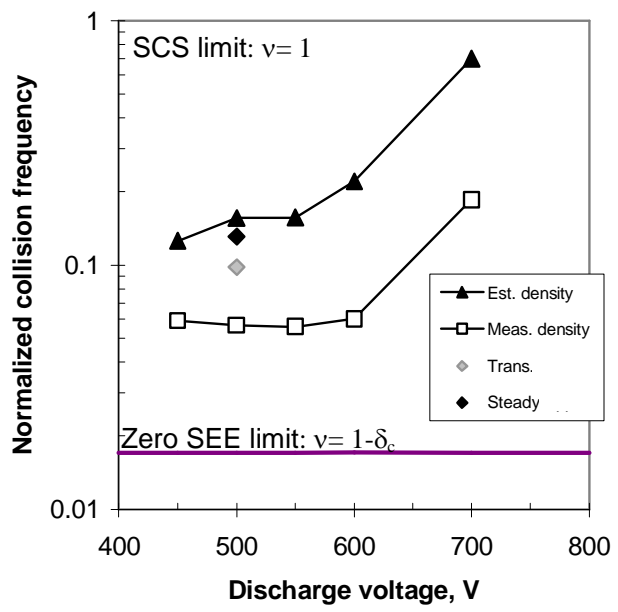

Figure 3. The dependence of the maximum electron temperature on the discharge voltage for different magnetic field and channel width configurations, and for both steady state and transitional operating regimes (a). The frequency ratio $^{7}$ (the experimental electron-wall collision frequency to the theoretical frequency) obtained for the thruster configuration shown in Fig. 1a in transitional regime (lines with markers) and for the thruster configuration shown in Fig. 1b (markers only) Here, $\delta_{\mathrm{c}} \approx 0.983$. For each thruster configuration, the magnetic field is not changed with the discharge voltage. The thruster channel is made of a grade HP boron nitride ceramic.

For the temperature saturation regime, by using the measured plasma parameters and exploiting the electron energy balance and Eq. (2), we obtained the experimental electron-wall collision frequency at the location of the maximum of the electron temperature. We compared this frequency with the theoretical frequency, given by Eq. (1). The comparison of these frequencies ${ }^{7}$ (Fig. 3b) suggests that the experimental electron-wall frequency for high discharge voltages is much lower than the theoretical value obtained for space charge saturated sheath regime, but larger than the wall recombination frequency.

We now describe the effects of the channel narrowing on the plasma parameters measured in the thruster with boron nitride channel walls (Figs. 4, 5). These measurements were conducted with the fixed magnetic field shown in Fig. 1b. The channel narrowing, which should enhance electron-wall collisions, causes unexpectedly larger changes of the plasma potential distribution than does the increase of the electron temperature with the discharge voltage. ${ }^{6}$ For the narrow channel configuration, the acceleration region is located almost completely in the near field plasma plume (Fig. 4). We found that this result holds for all discharge voltages used in these experiments (200-500 V), i.e., below and above the voltage threshold for the electron

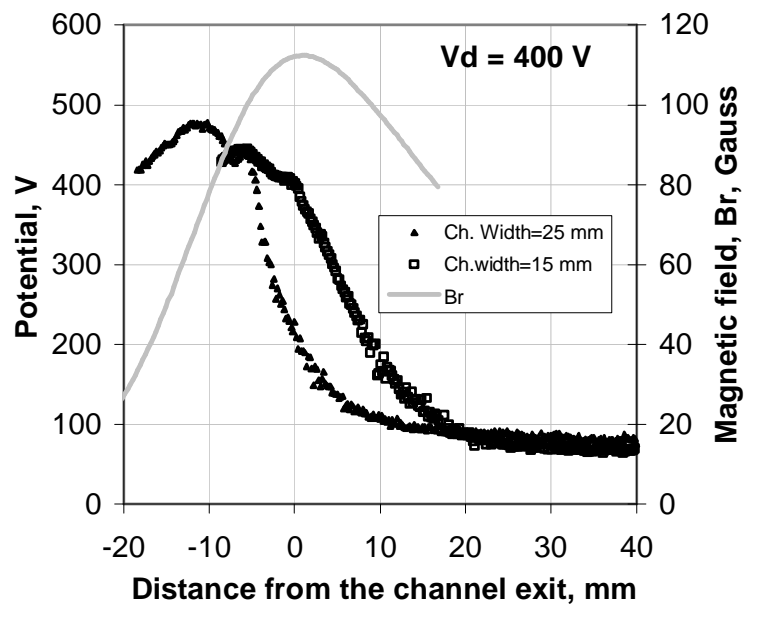

Figure 4. Plasma measurements for two thruster configurations (Fig. 1b) with different boron nitride channels of $25 \mathrm{~mm}$ width and $15 \mathrm{~mm}$ width. The anode location is $-\mathbf{4 6} \mathbf{~ m m}$. The discharge voltage is $400 \mathrm{~V}$ 
temperature saturation (Fig. 3a). The enhanced anomalous cross-field mobility (near-wall or Bohmtype) was suggested by a hydrodynamic model ${ }^{6}$ as an explanation to the reduced electric field measured inside the narrow channel. From our simulations described in Ref. 6, it appears that variation of the channel width only is insufficient to reproduce the experimental result. This reflects the fact that nearwall conductivity depends on the electron EDF, SEE etc. In addition, the reduced magnetic mirror near the inner wall (Fig. 1b) of the narrow channel might also affect the plasma potential distribution. The magnetic mirror near the inner wall may also affect electron $\mathrm{EDF}^{6}{ }^{6}$ and, therefore, also the electron-wall interaction, leading to the enhanced electron-wall collisions.

Having a larger potential drop in the near field plasma plume can have some advantages and disadvantages for the thruster applications. A possible advantage is that fewer energetic ions can strike the walls and cause the channel erosion. ${ }^{19}$ However, the ion acceleration occurs in the fringing magnetic field, where the plasma flow is divergent due to the defocusing equipotential surfaces. ${ }^{20}$ Within the accuracy of plume measurements, we obtained a less than $10 \%$ larger plume angle for the narrow channel, as compared with the wide channel (Fig. 5). Interestingly, as the discharge voltage increases above $600 \mathrm{~V}$, the difference in the plume angle diminishes, but the thruster with the narrow channel operates more efficiently in terms of the product of the current and propellant utilization efficiencies (Fig. 5).

\section{Conclusions}

We further demonstrated how the electron temperature and the plasma potential distribution can be affected by the discharge voltage and channel width. We found a large quantitative disagreement between the predictions of the existing theoretical models and the experimental results, in particularly, with respect to the maximum electron temperature attainable in Hall thrusters and the discharge voltage threshold for the electron temperature saturation. However, this disagreement does not necessarily imply that the electron temperature saturation measured for the conventional thruster with boron nitride channel walls is not associated with a strong SEE effects. In fact, the importance of SEE effects at high discharge voltages is evident from the absence of the temperature saturation for the thruster with low-SEE segmented electrodes. ${ }^{13}$ Therefore, the comparison of theoretical and experimental results demonstrates that the existing fluid models cannot quantitatively predict the dependence of the electron temperature on the discharge voltage and explain the electron temperature saturation which according to our measurements, ${ }^{7}$ is accompanied with a decrease of the Joule heating. Our experimental results indirectly support recent kinetic studies of Hall thrusters, ${ }^{21-24}$ which suggest that the electron-wall interaction in Hall thrusters is governed by kinetic effects (depleted electron $\mathrm{EDF}^{21-23}$, strong EDF anisotropy, ${ }^{24}$ beams of cold secondary electrons ${ }^{24}$ and non-stationary regimes $\left.^{24}\right)$.

We also showed that the channel narrowing significantly reduces the electric field inside the channel and causes the accelerating voltage drop to be established mainly in the near-field plasma plume. Enhanced anomalous cross-field mobility (near-wall or Bohm conductivity) is suggested by a hydrodynamic model ${ }^{6}$ as an explanation to the reduced electric field measured inside the narrow channel. A practical implication of the channel narrowing effect is that it may be used to reduce the channel erosion, which is more critical for high discharge voltage operation because of a larger energy of ions impinging the channel walls.

\section{Acknowledgments}

The authors wish to thank Dr. Leonid Dorf for his help with experiments and useful discussions. The authors benefited from discussions with Prof. Amnon Fruchtman, Dr. Igor Kaganovich, Mr. Dmitro Sydorenko and Prof. A. Smolyakov. This work was supported by US DOE Contract No. AC02-76CH0-3073. 


\section{References}

1. A. I. Morozov and V. V. Savel'ev, in Reviews of Plasma Physics, edited by B. B. Kadomtsev and V. D. Shafranov, ( Consultants Bureau, New York, 2000), Vol. 21, p. 203.

2. S. Barral, K. Makowski, Z. Peradzynski, N. Gascon and M. Dudeck, Phys. Plasmas 10, 4137 (2003).

3. E. Ahedo, J. M. Gallardo and M. Martinez-Sanchez, Phys. Plasmas 10, 3397 (2003).

4. E. Ahedo and D. Escobar, J. Appl. Phys. 96, 983 (2004).

5. G. J. M. Hagelaar, J. Bereilles, L. Garrigues and J.-P. Boeuf, J. Appl. Phys. 93, 67 (2003).

6. Y. Raitses, D. Staack, M. Keidar, N. J. Fisch, Phys. Plasmas 12, 057104 (2005).

7. Y. Raitses, D. Staack, A. Smirnov and N. J. Fisch, Phys. Plasmas 12, 073507 (2005).

8. J. M. Haas and A. D. Gallimore, Phys. Plasmas 8, 652 (2001).

9. D. Staack, Y. Raitses and N. J. Fisch, Appl. Phys. Lett. 84, 3028 (2004).

10. A. Smirnov, Y. Raitses, and N. J. Fisch, J. Appl. Phys. 94, 852 (2003).

11. M. Keidar, I. Boyd and I. I. Beilis, Phys. Plasmas 8, 5315 (2001).

12. G. D. Hobbs and J. A. Wesson, Plasma Phys. 9, 85 (1967).

13. Y. Raitses, D. Staack, and N. J. Fisch, submitted to J. Appl. Phys. (2005); Y. Raitses, A. Smirnov, D. Staack and N. J. Fisch, submitted to Phys. Plasmas (2005).

14. Y. Raitses, D. Staack, A. Dunaevsky, L. Dorf and N. J. Fisch, Proceedings of the $28^{\text {th }}$ International Electric Propulsion Conference, March 2003, Toulouse, France (Electric Rocket Propulsion Society, Cleveland, OH 2003), IEPC paper 2003-139.

15. D. Staack, Y. Raitses and N. J. Fisch, Rev. Sci. Instum. 75, 393 (2004).

16. G. S. Janes and J. P. Dotson, Rev. Sci. Instrum. 35, 1617 (1964).

17. R. R. Hofer, Ph. D Thesis, University of Michigan, 2004.

18. A. I. Morozov, A. I. Bugrova, A. V. Desyatskov, and V. K. Kharchevnikov, Plasma Phys. Rep. 22, 302 (1996).

19. L. Garrigues G. J. M. Hagelaar, J. Bereilles, C. Boniface and J.-P. Boeuf, Phys. Plasmas 10, 4886 (2003).

20. A. Fruchtman and A. Cohen-Zur, Proceedings of the $40^{\text {th }}$ Joint Propulsion Conference and Exhibit, July, 2004, Fort Lauderdale, FL (American Institute of Aeronautics and Astronautics, Reston, VA 2004), AIAA paper No. 2004-3957.

21. N. Meeazan and M. Cappelli, Phys. Rev. E 66, 036401 (2002).

22. A. Smirnov, Y. Raitses and N. J. Fisch, Phys. Plasmas 11, 4922 (2004).

23. O. Batishchev and M. Martinez-Sanchez, Proceedings of the $28^{\text {th }}$ International Electric Propulsion Conference, March 2003, Toulouse, France (Electric Rocket Propulsion Society, Cleveland, OH 2003), IEPC paper 2003-188.

24. D. Sydorenko and V. Smolyakov, Bull. Am. Phys. Soc. 49, NM2B008 (2004). 